\title{
Evaluation of real definite Integrals by using mixed quadrature rules over a Triangular Domain
}

\author{
${ }^{1}$ Pritikanta Patra , ${ }^{2}$ Dwitikrushna Behera,${ }^{3}$ Rajani Ballav Dash \\ Department of Mathematics, Ravenshaw University, Cuttack-753003, Odisha, India \\ 1pritikanta@yahoo.com \\ Department of Mathematics, Ravenshaw University, Cuttack-753003, Odisha, India \\ 2 dwiti78@gmail.com \\ Department of Mathematics, Ravenshaw University, Cuttack-753003, Odisha, India \\ 3 rajani_bdash@rediffmail.com
}

\begin{abstract}
A mixed quadrature rule of higher precision for approximate evaluation of real definite integrals over a triangular domain has been constructed. The relative efficiencies of the proposed mixed quadrature rules have been verified by using suitable test integrals. In this paper,we present a mixed quadrature i.e. mixed quadrature of anti-Lobatto rule and Fejer's first rule in one variable. For real definite integral over the triangular surface: $\{(x, y) \mid 0 \leq x, y \leq 1, x+y \leq 1\}$ in the Cartesian two-dimensional $(x, y)$ space,mathematical transformation from $(x, y)$ space to $(\xi, \eta)$ space maps the standard triangle in $(x, y)$ space to a standard 2-square in $(\xi, \eta)$ space: $\{(\xi, \eta) \mid-1 \leq \xi, \eta \leq 1\}$.
\end{abstract}

\section{Indexing terms/Keywords}

Anti-Lobatto four point rule; Fejer's first rule; mixed quadrature rule; finite element method(FEM); triangular elements; standard 2-square;extended numerical integration.

\section{Academic Discipline And Sub-Disciplines}

Numerical Method,Numerical Quadrature

\section{SUBJECT CLASSIFICATION}

2000 Mathematics Subject Classification: 65D30, 65D32

\section{TYPE (METHOD/APPROACH)}

Mixed quadrature

\section{INTRODUCTION}

In science and engineering, we observe that some integral problems can't be easily evaluated analytically but it is possible to calculate in numerically.So that numerical techniques are very good tool for solving different integral problems. This paper describes a method for the evaluation of integrals over a triangle using mixed quadrature rules. In particular, they are used for problems involving calculation of mass shell, fluid and mass flows across a surface, electric charge distribution over a surface, plate bending and heat conduction over a plate. The basic problem of integration of an arbitrary function of two variables over the surface of a triangle was first introduced by Hammer et. al. $[1,2,3]$. In connection of FEM, the triangular elements provide tremendous results. Cowper [4] provided a table of Gaussian quadrature formula for symmetrically placed integration points. Lyness and Jespersen [5] made an elaborate study of symmetric quadrature rules by formulating the problem in polar coordinates. Lannoy [6] discussed the numerical error in integration rule [4]. Laurie [7] derived 7-point integration rule and discussed the numerical error in integrating some functions. Laursen and Gellert [8] gave a detailed table of symmetric integration formulae and suggested some new higher-order formulae of precision up to degree 10. Lether [9], Hillion[10] and Lague and Baldur [11] considered the product formulae derivable from onedimensional Gaussian quadrature rules. Reddy [12 ], and Reddy and Shippy [14]derived 3-, 4-, 6 and 7-point formulae which give improved accuracy. The formulation of mixed quadrature rules was first coined by R. N. Das and G. Pradhan [15]. D. P. Laurie [21] is first to coin the idea of anti-Gaussian quadrature formula. An anti-Gaussian quadrature formula is $(n+1)$ point formula of degree $(2 n-1)$ which integrates all polynomials of degree up to $(2 n+1)$ with an error equal in magnitude but opposite in sign to that of $n$-point Gaussian formula.Many authors $[16,17,19]$ have produced different mixed quadrature rules.

In this paper, we get motivation for successfully forming a mixed quadrature rule of anti-Lobatto rule and Fejer's first rule over a triangular domain. The mixed quadrature rule over a triangular domain so found has been tested and compared with its constituent rules by computing numerically three test integrals.

\section{FORMULATION OF INTEGRALS OVER A TRANGULAR AREA}


The numerical integration of an arbitrary function $f$ over the triangle $T$ is given by

$$
I=\iint_{T} f(x, y) d x d y=\int_{0}^{1} d x \int_{0}^{1-x} f(x, y) d y=\int_{0}^{1} d y \int_{0}^{1-y} f(x, y) d x .
$$

It is now required to find the value of the integral by a quadrature formula:

$$
\mathrm{I}=\sum_{m=1}^{N} C_{m} f\left(x_{m}, y_{m}\right) \text {. }
$$

Where $C_{m}$ are the weights associated with specific points $\left(x_{m}, y_{m}\right)$ and $N$ is the number of pivotal points related to the required precision.

The double integral over the triangle surface of equation (2.1) can be transformed to the standard square $\{(u, v) \mid 0 \leq u \leq 1,0 \leq v \leq 1\}$ by substitution $x=u \quad$ and $y=(1-u) v$, we have $I=\int_{0}^{1} \int_{0}^{1-x} f(x, y) d y d x=\int_{0}^{1} \int_{0}^{1} f(x(u, v), y(u, v)) J d u d v$.

$$
\text { Where } J(u, v)=\left|\begin{array}{ll}
\frac{\partial x}{\partial u} & \frac{\partial y}{\partial u} \\
\frac{\partial x}{\partial v} & \frac{\partial y}{\partial v}
\end{array}\right|=1-u \text {. }
$$

From equation (2.3), we have $I=\int_{0}^{1} \int_{0}^{1} f(u,(1-u) v)(1-u) d u d v$.

The integral $I$ of equation (2.4) can be transformed further into an integral over the standard 2-square: $\{(\xi, \eta) \mid-1 \leq \xi \leq 1,-1 \leq \eta \leq 1\}$ by substitution $u=\frac{1+\xi}{2}, v=\frac{1+\eta}{2}$.

Then clearly the determinant of the Jacobian and the differential area are

$$
\begin{gathered}
\frac{\partial(w, v)}{\partial(\xi, v)}=\frac{\partial u}{\partial \xi} \frac{\partial v}{\partial \eta}-\frac{\partial u}{\partial \eta} \frac{\partial v}{\partial \xi}=\frac{1}{2}\left(\frac{1}{2}\right)-0 \times 0=\frac{1}{4} \\
d u d v=\frac{\partial(u, v)}{\partial(\xi, \eta)} d \xi d \eta=\frac{1}{4} d \xi d \eta
\end{gathered}
$$

Now on using equations (2.5) and (2.6) in equation(2.4) we have

$$
\begin{aligned}
I=\int_{0}^{1} \int_{0}^{1-x} f(x, y) d y d x & =\int_{0}^{1} \int_{0}^{1} f(u,(1-u) v)(1-u) d u d v \\
= & \int_{-1}^{1} \int_{-1}^{1} f\left(\frac{1+\xi}{2}, \frac{(1-\xi)(1+\eta)}{4}\right)\left(\frac{1-\xi}{8}\right) d \xi d \eta
\end{aligned}
$$

Equation(2.7) represents an integral over the surface of standard 2-square:

$\{(\xi, \eta) \mid-1 \leq \xi \leq 1,-1 \leq \eta \leq 1\}$. Efficiently quadrature coefficients are readily obtained from [20].

From equation(2.7), we can write:

$$
\begin{gathered}
I=\int_{-1}^{1} \int_{-1}^{1} f(x(\xi, \eta), y(\xi, \eta))\left(\frac{1-\xi}{8}\right) d \xi d \eta \\
I \cong \sum_{i=1}^{n} \sum_{j=1}^{n}\left(\frac{1-\xi_{i}}{8}\right) w_{i} w_{j} f\left(x\left(\xi_{i}, \eta_{j}\right), y\left(\xi_{i}, \eta_{j}\right)\right) .
\end{gathered}
$$

Where $\xi_{i}, \eta_{j}$ are Gaussian points in the $\xi, \eta$ directions respectively, and $w_{i}, w_{j}$ are the corresponding weights.

We can write equation(2.8) as:

$$
I \cong \sum_{k=1}^{N=n \times n} C_{k} f\left(x_{k v} y_{k}\right)
$$

Where $C_{k}, x_{k v} y_{k}$ are obtained from the relation 


$$
\left.\begin{array}{c}
c_{k}=\left(\frac{1-\xi_{i}}{8}\right) w_{i} w_{j} \\
x_{k}=\frac{1+\xi_{i}}{2} \\
y_{k}=\frac{\left(1-\xi_{j}\right)\left(1+\eta_{j}\right)}{4}
\end{array}\right\}
$$

Where $k=1,2, \ldots, n$

$$
\begin{aligned}
& i=1,2, \ldots, n \\
& j=1,2, \ldots, n
\end{aligned}
$$

The weighting coefficients $C_{k}$ and sampling points $\left(x_{k v} y_{k}\right)$ of various orders can now be easily computed by formulae (2.9) and (2.10). We have tabulated a sample of these weight coefficients and sampling points in Table-1.

\section{MIXED QUADRATURE OF ANTI-LOBATTO RULE AND FEJER'S $1^{\text {ST }}$ RULE IN ONE VARIABLE}

The mixed quadrature $\left(I_{\text {mix }}\right)$ of Anti-Lobatto rule (which has been framed by us in the light of anti-Gaussian rule ) and Fejer 's first rule is given below:

$$
\begin{aligned}
I_{\text {mix }} & =\int_{-1}^{1} f(x) d x \\
& \approx \frac{30}{99}\left[f\left(\sqrt{\frac{2}{5}}\right)+f\left(-\sqrt{\frac{2}{5}}\right)\right]-\frac{3}{99}[f(-1)+f(1)]+\frac{32}{99}\left[f\left(\frac{\sqrt{3}}{2}\right)+f\left(-\frac{\sqrt{3}}{2}\right)\right]+\frac{80}{99} f(0) .
\end{aligned}
$$

Where $w_{1}=\frac{30}{99}, w_{2}=\frac{30}{99}, w_{3}=-\frac{3}{99}, w_{4}=-\frac{3}{99}, w_{5}=\frac{32}{99}, w_{6}=\frac{32}{99}, w_{7}=\frac{80}{99}$.

Applying the mixed rule(3.1) to double integral

$$
I_{\text {mix }}=\int_{-1}^{1} \int_{-1}^{1} f(x(\xi, \eta), y(\xi, \eta))\left(\frac{1-\xi}{8}\right) d \xi d \eta
$$

we have

$$
I_{\text {mix }} \approx \sum_{k=1}^{49} C_{k} f\left(x_{i j}, y_{i j}\right)=\sum_{k=1}^{49} C_{k} f\left(x_{k}, y_{k}\right)
$$

Where $C_{k}=C_{i j}=\left(\frac{1-\xi_{i}}{8}\right) w_{i} w_{j}$ \}

$$
x_{k}=x_{i j} \text { and } y_{k}=y_{i j} \text { ) }
$$

The weighting coefficients $C_{k}$ and sampling points $\left(x_{k}, y_{k}\right)$ of various orders can be easily computed using the equation (2.10).

\section{NUMERICAL VERIFICATIONS}

\begin{tabular}{|l|l|l|l|}
\hline Integrals & Exact Value & Approximate Values & Error| \\
\hline$I_{1}=\int_{0}^{1} \int_{0}^{1-y}(x+y)^{\frac{1}{2}} d x d y$ & 0.400000000 & 0.401243 & 0.001243 \\
$I_{2}=\int_{0}^{1} \int_{0}^{1-y}(x+y)^{-\frac{1}{2}} d x d y$ & 0.666666667 & 0.641119 & \\
\hline$I_{a}=\int_{0}^{1} \int_{0}^{1-x} e^{-y^{2}} \cos (x y) d x d y$ & 0.4284998849 & 0.429191 & 0.025547667 \\
\hline
\end{tabular}

\section{CONCLUSIONS}


From the numerical verification we conclude that the mixed quadrature rule used in this paper gives better result than those obtained in the previous papers $[13,18,19]$ on integration of real functions over triangles.

\section{REFERENCES}

[1] P. C. Hammer, O. J. Marlowe and A. H. Stroud, "Numerical integration over simplexes and cones", Math Tables other Aids Computation, 10, 130-136 (1956).

[2] P. C. Hammer and A. H. Stroud, "Numerical integration over simplexes", Math Tables other Aids Computation, 10, 137-139 (1956).

[3] P. C. Hammer and A. H. Stroud, "Numerical evaluation of multiple integrals", Math Tables other Aids Computation, 12, 272-280 (1958).

[4] G. R. Cowper, "Gaussian quadrature formulas for triangles”, Int. Num. Meth. Engg., 7, 405-408 (1973).

[5] J. N. Lyness and D. Jespersen, "Moderate degree symmetric quadrature rules for the triangle", J. Inst. Math. Applic., 15, 19-32 (1975).

[6] F. G. Lannoy, "Triangular Finite Elements and Numerical Integration”, Computer Struct, 7, 613 (1977).

[7] D. P. Laurie, "Automatic numerical integration over a triangle", CSIR Spec. Rep. WISK 273, National Institute for Mathematical Sciences, Pretoria (1977).

[8] M. E. Laursen and M. Gellert, "Some criteria for numerically integrated matrices and quadrature formulas for triangles", Int. J. Num. Meth. Engg, 12, 67-76 (1978).

[9] F. G. Lether, "Computation of double integrals over a triangle”, J. Comp. Applic. Math., 2, 219-224 (1976).

[10] P. Hillion, "Numerical integration on a triangle", Int. J. Num. Meth. Engg., 11, 797-815 (1977).

[11] G. Lague and R. Baldur, "Extended numerical integration method for triangular surfaces", Int. J. Num. Meth. Engg.", 11, 388-392 (1977).

[12] C. T. Reddy, "Improved three point integration schemes for triangular finite elements", Int. J. Num. Meth. Engg.", 12, 1890-1896 (1978).

[13] H. T. Rathod et. al., "Gauss-Legendre Quadrature over Triangles", J. Indian Inst. Sci., 84, 183 188(2004).

[14] C. T. Reddy and D. J. Shippy, "Alternative integration formulae for triangular finite elements", Int. J. Num. Meth. Engg.", 17, 133-139(1981).

[15] R. N. Das and G. Pradhan, "A mixed quadrature rule for approximate evalution of real definite integrals", Int. J. Math. Educ. Sci. and Technology, 27(2), 279-283 (1996).

[16] D. K. Behera, A. K. Sethi and R. B. Dash, "An Open type Mixed Quadrature Rule using Fejer and Gaussian Quadrature Rules”, American International Journal of Research in Science, Technology, Engineering \& Mathematics", 9(3), 265-268 (2015).

[17] D. K. Behera and R. B. Dash, "A Mixed Quadrature Rule for Numerical Integration of Analytic Functions by using Fejer and Gaussian Quadrature Rules", Bulletin of Pure and Applied Sciences, 34E(1 2), 61-67 (2015).

[18] K. T. Shivaram, “Generalised Gaussian Quadrature over a triangle”, American Journal of Engineering Research, 02(09), 290-293(20130.

[19] S. R. Jena and R. B. Dash, "Mixed Quadrature of real definite Integral over triangles", Pacific-Asian Journal of Mathematics, 3(1-2), 119-124(2009).

[20] M. Abramowicz and I. A. Stegum(eds), Handbook of Mathematical Functions, Dover (1964).

[21] D. P. Laurie, Anti-Gaussian Quadrature formulas, Mathematics of Computation, 65, 739-749(1996). 